\title{
Anisotropic rare-earth spin-glasses
}

\author{
A. Fert, P. Pureur, and F. Hippert \\ Laboratoire de Physique des Solides, Université de Paris-Sud, F-91405 Orsay, France \\ K. Baberschke and F. Bruss* \\ Institut für Atom and Festkörperphysik, Freie Universität Berlin, Boltzmannstrasse 20, D-1000 Berlin 33, Germany
}

(Received 6 July 1982)

\begin{abstract}
We present magnetic measurements (ac and dc susceptibilities and magnetization) on $Y \mathrm{Er}$ and $Y \mathrm{Gd}$ single crystals. The longitudinal (i.e., along the $c$ axis) susceptibility of $Y$ Er shows a typical spin-glass-like cusp, whereas no cusp is observed in the transverse directions. This suggests that only the longitudinal spin components are frozen, in agreement with the theoretical predictions of Cragg and Sherrington for a spin-glass with a large uniaxial crystal field. The $Y \mathrm{Er}$ system appears to represent an almost perfect Ising type of spin-glass. Surprisingly the $Y$ Gd system is also definitely anisotropic. A spin-glass-like cusp is observed only for the transverse susceptibility. This anisotropy cannot be ascribed to the crystal field and we discuss its possible origins. Finally, we present some data on the remanence properties of the $Y \mathrm{Er}$ and $Y \mathrm{Gd}$ alloys.
\end{abstract}

\section{INTRODUCTION}

The properties of the spin-glass state have been extensively studied in cubic systems and rarely in crystals of uniaxial symmetry. However, studies in uniaxial spin-glass systems should be of great interest because, depending on the sign and the magnitude of the uniaxial crystal field acting on the magnetic ma- ments $\left\{\right.$ i.e., $\left.H_{\mathrm{CF}}=B_{20} O_{20} \equiv B_{20}\left[3 J_{z}^{2}-J .(J+1)\right]\right\}$ these systems can be Ising-like (for $B_{20}$ negative and much larger than $k T_{g}$ ), $X Y$-like (for $B_{20}$ positive and much larger than $k T_{g}$ ) or Heisenberg-like (for $B_{20}$ much smaller than $k T_{g}$ ).

Some theoretical aspects of these situations have been developed by Ghatak and Sherrington ${ }^{1}$ a few years ago. More recently, Cragg and Sheerington ${ }^{2}$ have extended the Sherrington-Kirkpatrick (SK) model $^{3}$ of spin-glasses to the case of a system of spins in a uniaxial crystal field. In particular, this theory predicts spin-glass ordering for the longitudinal (i.e., parallel to the crystal-field axis) spin components and not for the transverse ones when the crystal-field constant $B_{20}$ is negative and large enough with respect to the exchange coupling.

Few experimental studies of uniaxial spin-glasses performed on single crystals exist. There have been investigations of $\left(\mathrm{Ti}_{1-x} \mathrm{~V}_{x}\right)_{2} \mathrm{O}_{3}$ (Ref. 4) and $\left(\mathrm{Ni}_{1-x} \mathrm{Fe}_{x}\right)_{1-\delta} \mathrm{S}$ (Ref. 5) single crystals revealing spin-glass-like maxima of the susceptibility only in certain lattice directions or different maxima in different lattice directions. However, these spin-glass systems are not very simple, the origin of the magnetic interactions is not well known in $\left(\mathrm{Ti}_{1-x} \mathrm{~V}_{x}\right)_{2} \mathrm{O}_{3}$, while in $\left(\mathrm{Ni}_{1-x} \mathrm{Fe}_{x}\right)_{1-\delta} \mathrm{S}$ it is supposed that the magnetic moments are developed on pairs of iron impurities. Recently, Albrecht et al. ${ }^{6}$ investigated the mag- netic properties of $Z n \mathrm{Mn}, C d \mathrm{Mn}$, and $M g \mathrm{Mn}$ single crystals. $Z n M n$ shows an Ising-like behavior with a spin-glass maximum of the susceptibility only for longitudinal fields, $C d \mathrm{Mn}$ shows a $X Y$-like behavior and $M g \mathrm{Mn}$ present isotropic properties. In this paper we report on an investigation of the spin-glass properties of $Y \mathrm{Er}$ and $Y \mathrm{Gd}$ single crystals. ${ }^{7} \mathrm{Up}$ to now the magnetic properties of yttrium-rare-earth alloys have been investigated only in polycrystals. ${ }^{8-10} \mathrm{~A}$ spin-glass behavior has been identified at low concentrations and a transition to helical ordering has been suggested at higher concentrations (above 2.8 at. \% in $Y \mathrm{Gd}$, above 5 at. $\%$ in $Y \mathrm{~Tb}){ }^{9}$

\section{II. $Y$ Er ALLOYS}

We first focus on the $Y$ Er system. Its crystal-field parameters have been determined by Touborg ${ }^{11}$ from measurements of the paramagnetic susceptibility of very dilute alloys and neutron scattering experiments. They are

$$
\begin{aligned}
& B_{20}=-2.8 \times 10^{-1} \mathrm{~K}, \quad B_{40}=6.0 \times 10^{-4} \mathrm{~K}, \\
& B_{60}=2.48 \times 10^{-5} \mathrm{~K}, \quad B_{66}=2.76 \times 10^{-4} \mathrm{~K} .
\end{aligned}
$$

The coefficients $B_{40}, B_{60}$, and $B_{66}$ are much smaller than $B_{20}$. If they could be neglected, the ground state would be the doublet $\left|\frac{15}{2}, \pm \frac{15}{2}\right\rangle$ and the system would be Ising-like in the low-temperature limit. When the coefficients $B_{40}, B_{60}$, and $B_{66}$ are taken into account, the actual ground state corresponds to the following combination ${ }^{11}$ :

$$
0.966\left|\frac{15}{2}, \pm \frac{13}{2}\right\rangle-0.228\left|\frac{15}{2}, \pm \frac{1}{2}\right\rangle+0.122\left|\frac{15}{2}, \mp \frac{11}{2}\right\rangle \text {. }
$$


This ground state is predominantly a $\left|\frac{15}{2}, \pm \frac{13}{2}\right\rangle$ state with a small admixture of $\left|\frac{15}{2}, \pm \frac{1}{2}\right\rangle$ and $\left|\frac{15}{2}, \pm \frac{11}{2}\right\rangle$ states. It lies at $27 \mathrm{~K}$ below the lowest of the other eigenstates and the overall splitting by crystal fields amounts to $123 \mathrm{~K}$. In spite of the complications arising from the fourth- and sixth-order terms, the oneion behavior observed in dilute alloys is not very different from what it would be with only quadratic terms, i.e., the one-ion susceptibility at low temperatures is much larger in longitudinal fields ( $\overrightarrow{\mathrm{H}}$ along $c$ ) than in transverse fields ( $\overrightarrow{\mathrm{H}}$ in the basal plane). ${ }^{11}$ Equally, the transverse susceptibility is almost isotropic when the magnetic field rotates in the basal plane. ${ }^{11}$ Thus we believe that it is reasonable, in first approximation, to forget the effects of the fourthand sixth-order terms and to consider that the $Y \mathrm{Er}$ system can be used to test the predictions of the theoretical models of spin-glasses with uniaxial quadratic anisotropy. Because the ground-state isolation is relatively large $(\sim 27 \mathrm{~K})$, we can also anticipate that the $Y$ Er system is probably not very far from the Ising limit in the temperature range of the spin-glass

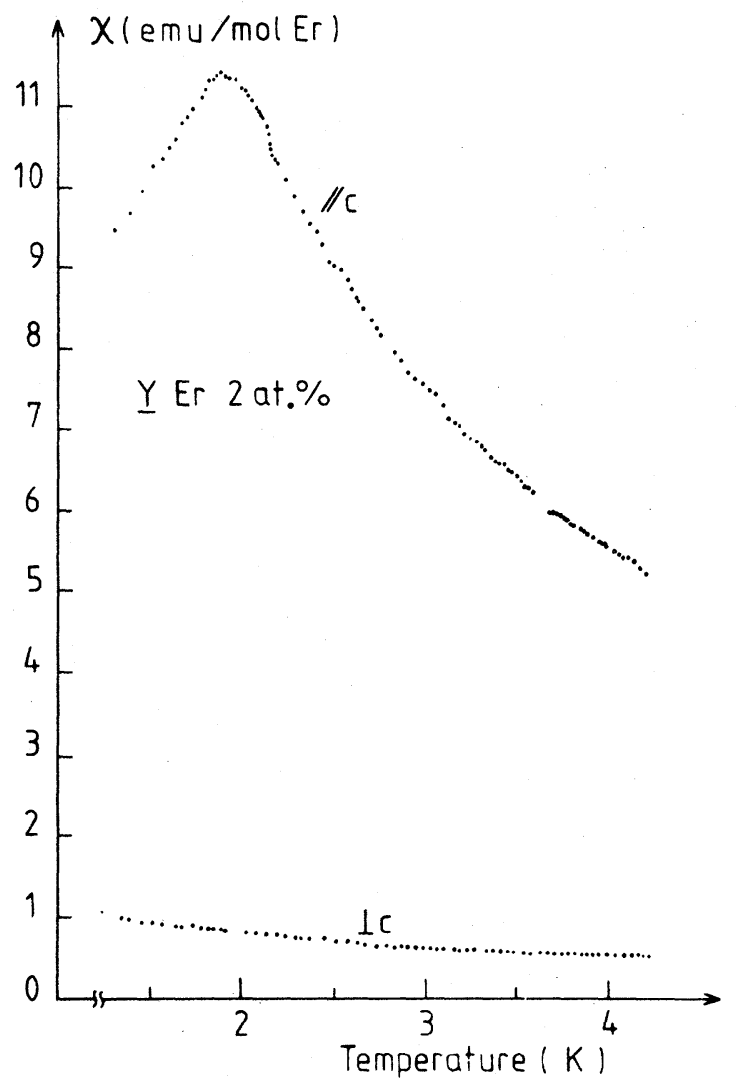

FIG. 1. ac magnetic susceptibility of $Y \operatorname{Er} 2$ at. \% for fields along the $c$ axis and along the $b$ axis (measurements along the $a$ axis give the same results as along the $b$ axis). The susceptibility of pure yttrium has been subtracted. The measurements have been performed in $1 \mathrm{G}$ at $120 \mathrm{~Hz}$.

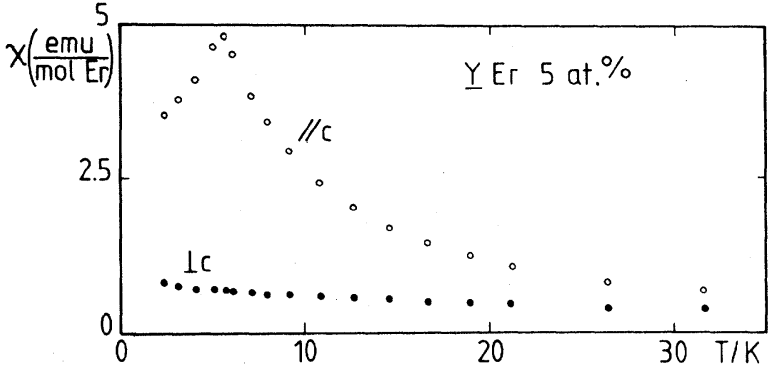

FIG. 2. dc magnetic susceptibility of $Y \operatorname{Er} 5$ at. \% measured with a vibrating sample magnetometer in $H=100 \mathrm{G}$ parallel or perpendicular to the $c$ axis.

state for moderate concentrations $\left(T_{g} \sim 1 \mathrm{~K} /\right.$ at. \%).

We show in Figs. 1 and 2 the longitudinal and transverse susceptibilities of two $Y \mathrm{Er}$ alloys as a function of temperature (the longitudinal or transverse susceptibilities of pure $Y$ have been subtracted). The longitudinal susceptibility shows a spin-glass typical cusp at $T_{g}=1.9 \mathrm{~K}$ for $c=2$ at. \% and $T_{g}=5.6 \mathrm{~K}$ for $c=5$ at. $\%$. In contrast, the transverse susceptibility increases continuously when the temperature decreases and there is no evidence of any event at $T_{g}$. As mentioned in our introduction, recent calculations ${ }^{2}$ predict spin-glass ordering only for the longitudinal-spin components when the crystal-field coefficient $B_{20}$ is negative and large enough with respect to the exchange interaction, while the transverse Edwards-Anderson parameter would remain zero down to $T=0$. Our experimental results, with a susceptibility cusp in longitudinal fields but not in transverse fields, should correspond to this situation. A similar behavior has been observed by Albrecht et al. ${ }^{6}$ in $\mathrm{ZnMn}$ alloys and by Roux-Buisson and Coey in some $\left(\mathrm{Ni}_{1-x} \mathrm{Fe}_{x}\right)_{1-\delta} \mathrm{S}$ alloys.

In longitudinal fields the $Y$ Er alloys exhibit the typical remanence properties of spin-glasses. We show in Fig. 3 the thermo-remanent and irreversible remanent magnetization (TRM and IRM) curves of

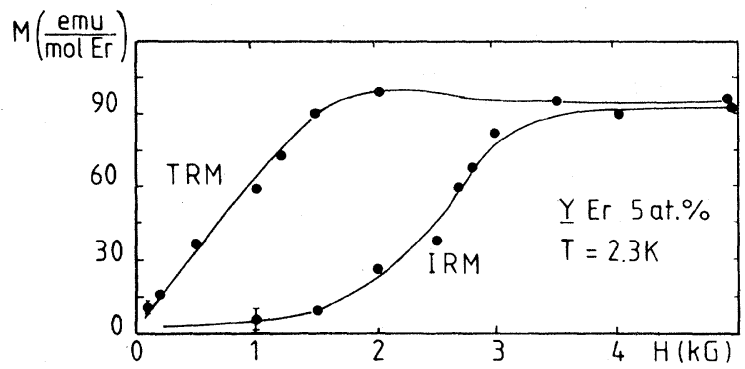

FIG. 3. Thermoremanent and irreversible remanent magnetization for a $Y \mathrm{Er} 5$ at. $\%$ crystal at $T=2.3 \mathrm{~K}\left(T / T_{g}=0.4\right)$ and for fields along the $c$ axis. The solid lines are guides for the eyes through the experimental points. 
$Y$ Er 5 at. \% These curves look like those observed in classical spin-glasses such as $C u \mathrm{Mn}$ or $A u \mathrm{Fe}$. However the magnitude of the remanent magnetization with respect to the reversible magnetization in the same fields is smaller than in $C u \mathrm{Mn}$ or $A u \mathrm{Fe}$. We could not observe any remanence effect in transverse fields.

\section{YGd SYSTEM}

The crystal field of the $Y \mathrm{Gd}$ system, in contrast to that of $Y \mathrm{Er}$, is very small. The coefficient $B_{20}$, derived from EPR data on dilute alloys by Weimann and Elschner, ${ }^{12}$ is

$$
B_{20}=-7.22 \times 10^{-3} \mathrm{~K} \text {. }
$$

This gives an overall crystal-field splitting of $0.26 \mathrm{~K}$. The effects of such a small crystal field on the spinglass properties can be anticipated to be negligible, at least in our alloys which have a freezing temperature higher than $2.1 \mathrm{~K}$. It can be noted, for example, that the crystal effects are negligible in the $M g \mathrm{Mn}$ alloys investigated by Albrecht et al., ${ }^{6}$ although the freezing temperature of these alloys is larger than their overall splitting by only a factor of 2 . However, our susceptibility measurements on $Y$ Gd single crystals show striking anisotropy effects. The susceptibility of $Y \mathrm{Gd}$ 1 at. \% (Fig. 4) and 2.3 at. \% (Fig. 5) show a typical spin-glass cusp for $\chi_{\perp}$ at $T=2.1$ and $7.0 \mathrm{~K}$, respec-

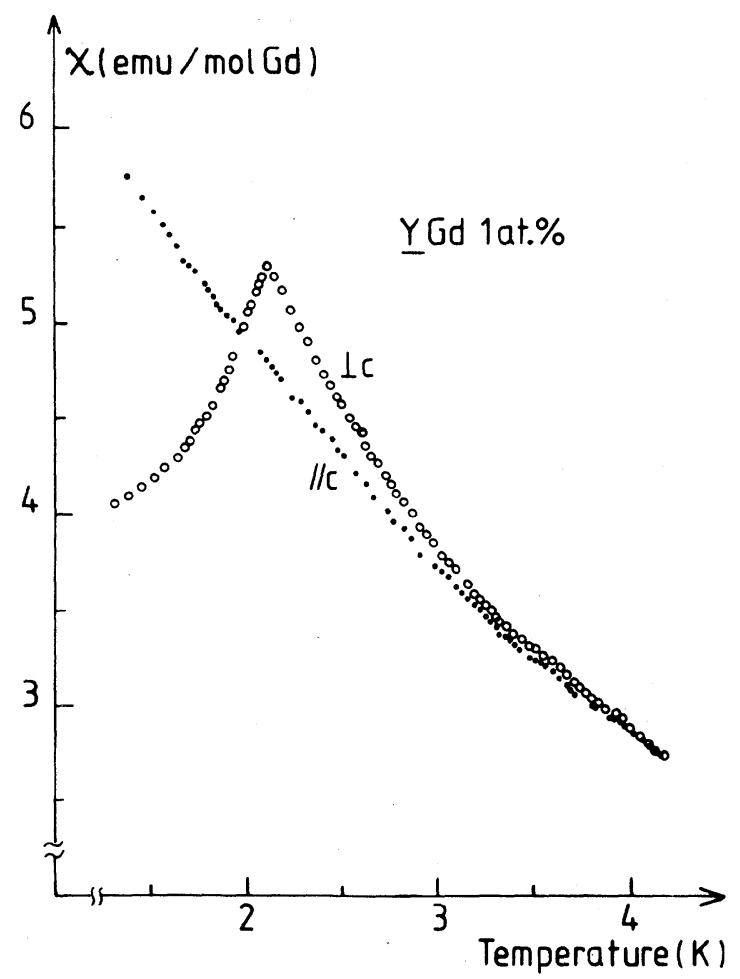

FIG. 4. Same caption as Fig. 1 but for $Y$ Gd 1 at. \%.

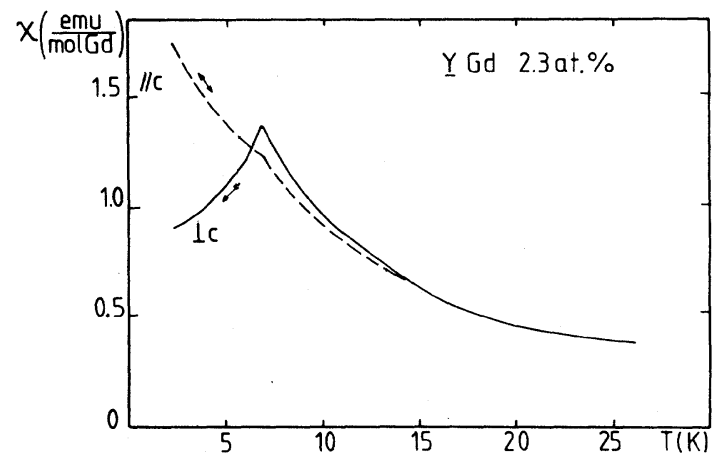

FIG. 5. de susceptibility of $Y \mathrm{Gd} 2.3$ at. \% in $H=100 \mathrm{G}$. As indicated by the arrows, there is no significant difference between FC and ZFC measurements. The small cusp in $\chi_{\|}$, which is not observed for $Y$ Gd 1 at. \%, could be due to a small misorientation of the crystal (admixture of a small transverse component).

tively. In contrast the longitudinal susceptibility monotonously increases for decreasing temperature. The susceptibility is isotropic at high temperatures (at least after subtracting the susceptibility of pure yttrium). When the temperature decreases, $\chi_{\perp}$ increases more rapidly than $\chi_{\|}$. Then, below $T_{g}, \chi_{\perp}$ decreases and becomes smaller than $\chi_{\|}$. These results are very striking. The anisotropy cannot be ascribed to the crystal field, first because the overall crystal-field splitting is very small, and then because, anyway, the negative sign of $B_{20}$ would lead to a larger longitudinal susceptibility above $T_{g}$. The concentration dependence of the anisotropy is also inconsistent with a crystal-field effect. Therefore, the anisotropy of the $Y$ Gd alloys must be ascribed to another sort of spinlattice coupling. A first possibility is a coupling of the Gd moments with spin-density waves in a conduction band with a significant spin-orbit coupling (a coupling with spin-density waves has been already proposed to account for a transition from spin-glass ordering to helical ordering at higher concentration $\left.^{8,9}\right)$. Another explanation could be the existence of anisotropic interactions between the Gd moments. More specifically the large spin-orbit coupling of the $5 d$ outer electrons of Gd should give rise to significant Dzyaloshinsky-Moriya-type interactions ${ }^{13}$ and, consequently, to a concentration-dependent pseudocrystal field.

Another definite difference between the $Y \mathrm{Er}$ and $Y$ Gd alloys concerns the remanence properties. The remanent magnetization of $Y \mathrm{Gd}$ is much smaller than that of $Y \mathrm{Er}$. As shown in Fig. 5 the dc susceptibility (magnetization at $100 \mathrm{G}$ ) is, within our experimental uncertainty, almost reversible and independent of field cooling (FC) or or zero field cooling (ZFC). We could not draw accurately TRM and IRM curves such as those of Fig. 3 for $Y$ Er. It turns out the yttrium-rare-earth system definitely departs from 
the classical transition-metal impurity spin-glasses. In the latter the remanence properties appear to be independent of the anisotropy properties; i.e., they are similar for alloys with weak spin-lattice couplings ( $C u \mathrm{Mn}, A g \mathrm{Mn}$ ) and for alloys with strong spin-lattice couplings $(A u \mathrm{Fe})$. In the yttrium-rare-earth system, the remanence properties seem to be associated with the existence of strong spin-lattice couplings ( $Y \mathrm{Er}$ ).

\section{CONCLUSIONS}

$Y$ Er: The existence of a cusp in the longitudinal susceptibility and not in the transverse seems to be in agreement with the calculations of Cragg and Sherrington, predicting spin-glass ordering only for longitudinal spin components in the presence of a strong enough uniaxial crystal field. The $Y \mathrm{Er}$ should be close to an Ising spin-glass system. On the other hand, $Y \mathrm{~Tb}$ or $Y \mathrm{Dy}$, for which the crystal-field coefficient $B_{20}$ is positive, should be close to the $X Y$-like spin-glass systems. Recent measurements have confirmed that $Y \mathrm{~Tb}$ shows a maximum of susceptibility only for transverse field. ${ }^{14}$

$Y \mathrm{Gd}$ : The properties of $Y \mathrm{Gd}$ exhibit a surprising anisotropy which cannot be ascribed to the crystal field (too small and having the wrong sign). The anisotropic spin-glass properties of $Y \mathrm{Gd}$ could be due to a coupling with spin-density waves in the conduction band of $Y$ or to anisotropic interactions between the $\mathrm{Gd}$ moments. We also found that the $Y \mathrm{Gd}$ alloys present very weak remanence effects.

Note added in proof. Theoretical results of Ref. 2 have also been found by S. A. Roberts and A. J. Bray, J. Phys. C $\underline{15}$, L527 (1982).

\section{ACKNOWLEDGMENT}

One of us (P.P.) was supported by Conselho National de Desenvolvimento Cientifico Tecnologico, Brasil.
"Now at Schoeller Elektronik D-3552 Wetter 1, Germany. 'S. K. Ghatak and D. Sherrington, J. Phys. C 10 , 3149 (1977).

${ }^{2}$ D. M. Cragg and D. Sherrington (unpublished).

${ }^{3}$ D. Sherrington and S. Kirkpatrick, Phys. Rev. Lett. $\underline{35}$, 1792 (1975).

4J. Dumas, C. Schlenker, J. L. Tholence, and R. Tournier, Phys. Rev. B 20, 3913 (1979).

${ }^{5}$ H. Roux-Buisson and J. M. D. Coey, J. Phys. F $\underline{10}, 275$ (1980).

${ }^{6}$ H. Albrecht, E. F. Wassermann, F. T. Hedgcock, and P. Monod, Phys. Rev. Lett. 48, 819 (1982).

${ }^{7}$ Part of the results have been communicated: F. Bruss and
K. Baberschke, Verh. Dtsch. Phys. Ges. 6, 186 (1979). We would like to thank Dr. Ghatak for discussions in the early state of the experiments.

${ }^{8}$ B. V. B. Sarkissian and B. R. Coles, Commun. Phys. 1,17 (1976).

${ }^{9}$ B. V. B. Sarkissian, J. Phys. F 7, L139 (1977).

${ }^{10}$ S. P. McAlister, J. Appl. Phys. 52, 1769 (1981).

11P. Touborg, Phys. Rev. B 16, 1201 (1977); P. Touborg, thesis (Odense University, 1979) (unpublished).

${ }^{12}$ G. Weimann and B. Elschner, Z. Phys. 261, 85 (1973).

${ }^{13}$ A. Fert and P. M. Levy, Phys. Rev. Lett. 44, 1538 (1981).

${ }^{14} \mathrm{P}$. Pureur and F. Hippert (private communication). 